\title{
Collaborative Engineering Paradigm Applied to the Aerospace Industry
}

\author{
Fernando Mas ${ }^{1}$, José Luis Menéndez ${ }^{1}$, Manuel Oliva ${ }^{1}$, \\ Alejandro Gómez ${ }^{2}$, and José Ríos ${ }^{2}$ \\ ${ }^{1}$ AIRBUS, Av. García Morato s/n, 41011, Sevilla, Spain \\ fernando.mas@airbus.com \\ ${ }^{2}$ Univ. Polit. Madrid, José Gutiérrez Abascal 2, 28006 Madrid, Spain \\ jose.rios@upm.es
}

\begin{abstract}
Airbus designs and industrializes aircrafts using Concurrent Engineering techniques since decades. The introduction of new PLM methods, procedures and tools, and the need to reduce time-to-market, led Airbus Military to pursue new working methods. Traditional Engineering works sequentially. Concurrent Engineering basically overlaps tasks between teams. Collaborative Engineering promotes teamwork to develop product, processes and resources from the conceptual phase to the start of the serial production. The CALIPSOneo pilot project was launched to support the industrialization process of a medium size aerostructure. The aim is to implement the industrial Digital Mock-Up (iDMU) concept and its exploitation to create shop floor documentation. In a framework of a collaborative engineering strategy, the project is part of the efforts to deploy Digital Manufacturing as a key technology for the industrialization of aircraft assembly lines. This paper presents the context, the conceptual approach and the methodology adopted.
\end{abstract}

Keywords: collaborative engineering, industrial Digital Mock-Up, PLM systems.

\section{Introduction}

The design and the industrialization of aircrafts is a complex process. An aircraft like the Airbus A400M is composed of about 700.000 parts (excluding standard parts). The parts are assembled into aerostructures and major components, which are designed and manufactured in seven countries all over the world [1].

In the mid-nineties, PDM and CAD systems let Airbus to build a product Digital Mock-Up (DMU), mainly to check functional design interferences. Then the ACE (Airbus Concurrent Engineering) project [2] started to develop and deploy methods, processes and tools along all the functional design disciplines. A summary of the Airbus product DMU is presented in [3].

Since 1999, Airbus has successfully applied Concurrent Engineering to all the aircraft design programs: A380, C295, A400M, and A350. The gain is obtained mainly in the functional design area. The Industrial design activities make use of CAx tools 
in not fully integrated areas, causing interoperability issues and a lack of real influence on the functional design.

The development of computer based shared workspaces was the main aim in the early years of the Collaborative Engineering concept and product modeling, or product DMU, was the main focus [4]. Currently, Collaborative Engineering promotes to integrate design teams, both functional and industrial, to generate a unique deliverable, named industrial DMU (iDMU), integrating: product, processes and resources. It also promotes the intensive use of PLM tools to perform virtual manufacturing and support the DMU as kernel of the collaborative work [5]. The downward link with the physical manufacturing is created by feeding work instructions (WIs) to the shop floor. Since the iDMU comprises the full definition of the manufacturing activities, it can be used to generate WIs [6].

In this context, Airbus Military launched the CALIPSOneo pilot project to contribute to develop and deploy Collaborative Engineering processes and tools. The project focuses on the new A320neo Fan Cowls. The low complexity of such aerostructure, the manufacturing technologies involved and the company expertise on the design of fairings make it an excellent sample to conduct a pilot project.

\section{Review of the Traditional, Concurrent and Collaborative Process}

The evolution inside Airbus from Traditional Engineering to Collaborative Engineering through Concurrent Engineering is an ongoing process. The main triggers are: technological evolution of software tools, the need to short time-to-market, to reduce cost and to increase quality and the maturity of the development team.

\section{Traditional Engineering}

The Traditional Engineering approach, also known as sequential engineering, comprises the implementation of sequential tasks, often referred to as the 'over-the-wall' approach [7]. Disadvantages of this approach are extensively mentioned in the literature: focus on product functionality and drawings, lack of industrial design, problems growing at the end of the lifecycle, different teams with lack of communication between them and long time-to-market.

\section{Concurrent Engineering}

In the last decades, methodological and technological advances have significantly influenced engineering activities. Concurrent Engineering has become widely accepted. About twenty years ago, with the introduction of the emergent PLM tools, a company-wide project, ACE (Airbus Concurrent Engineering) [2] was launched. Framed within the Airbus A340-500/600 development program, it aimed to develop and deploy concurrent engineering practices and the associated PLM tools. 

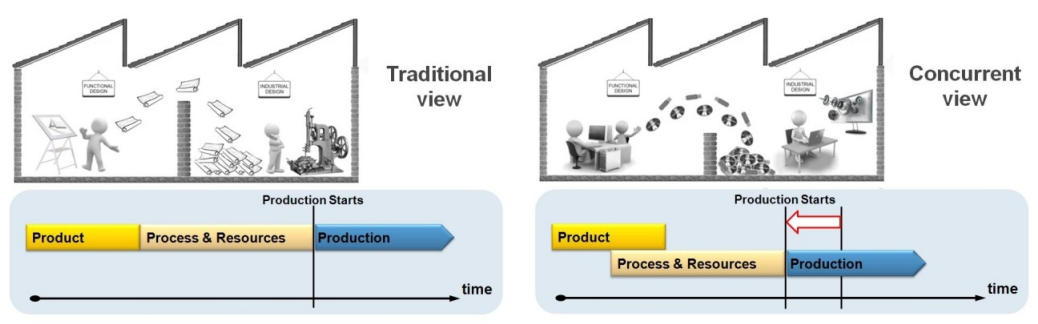

Fig. 1. Traditional view vs. Concurrent view

Currently, there are still pending issues, the wall described in Traditional Engineering still exits but it is not so high. The industrialization tasks are not as advanced as functional tasks in terms of PLM tools usage. The current deliverable is the product DMU, interoperability and working practice issues cause that compact disks or memory sticks flies over the wall instead of paper based drawings. Industrial design is not fully integrated with functional design, and it has little influence over the latter. They comprise two separate teams with dissimilar skills.

In fact, one of the most important reasons to have the wall is the certification process by the aeronautical authorities. Traditionally certification was made using the product definition, drawings, and marked the end of the aircraft design process. Concurrent Engineering still hold this idea and consider the aircraft design only as the functional design, enriched with manufacturing constrains and needs.

\section{Collaborative Engineering}

The iDMU is the key element of the Collaborative Engineering to tear down the wall between functional design and industrial design. The aim is a design process with a single team that creates a single deliverable. This approach needs new working procedures and new PLM tools. By applying virtual validation, using virtual manufacturing techniques, a further reduction of time-to-market is feasible.
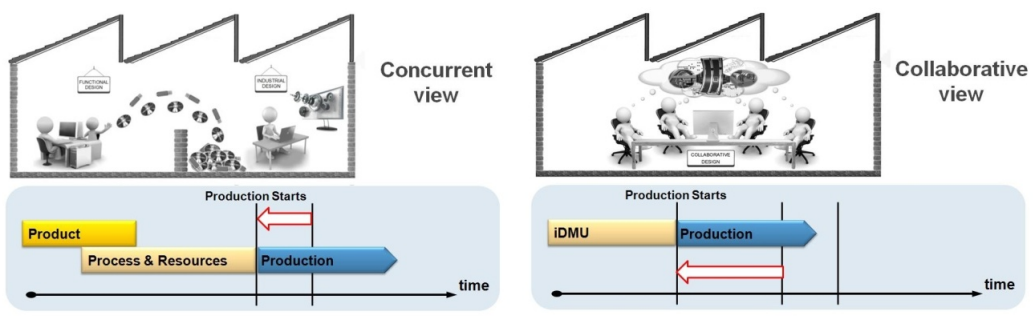

Fig. 2. Concurrent view vs. Collaborative view 


\section{Collaborative Engineering Airbus Military Conceptual Model}

Both the functional and the industrial design areas shared the concept of Collaborative Engineering. It is seen as an answer to the current industrial challenges.

\section{Analysis As-Is To-Be}

Current or 'As Is' situation (Fig. 3) shows an optimized functional design area with a clear deliverable: the product DMU. The concurrent process closes the gap between functional design and industrial design, and feeds back functional design with manufacturing information to facilitate 'Design for Manufacturing' and 'Design for Assembly'. The functional design deliverable, the 'DMU as master', is a valuable item in the first stages of the lifecycle, but the value for the process decreases with time and most of the industrial design tasks are paper based.

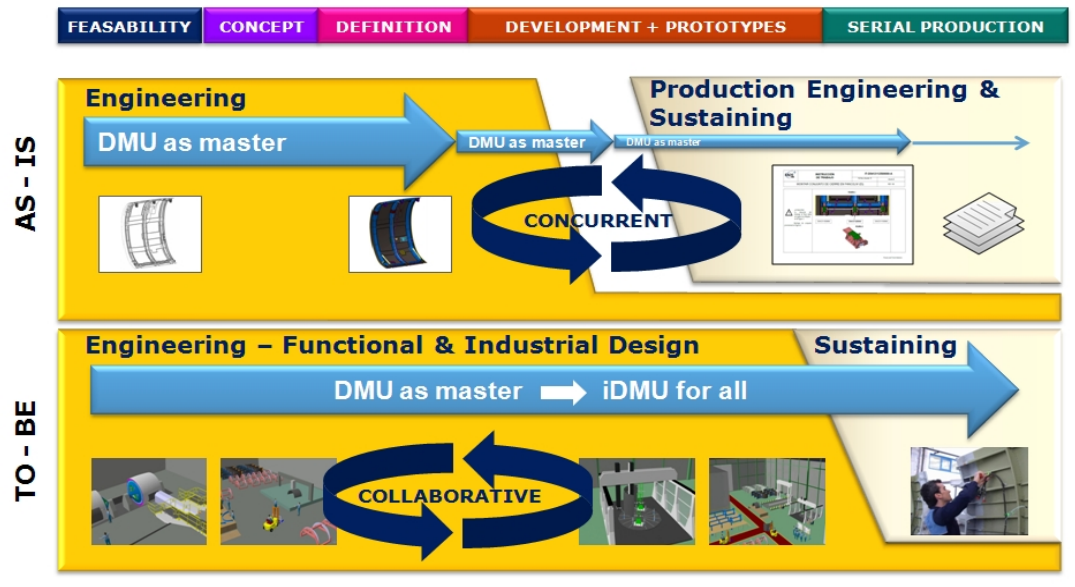

Fig. 3. Analysis 'As Is - To Be'

Future or 'To Be' situation shows an optimized functional and industrial design area with a clear deliverable: the iDMU [8]. The previous gap is eliminated by the collaborative way to build the iDMU and the virtual validation of it. The design (functional and industrial) deliverable, the 'iDMU for all', is a valuable item along the whole lifecycle. The information contained in the iDMU can now be exploited by Sustaining, to produce shopfloor documentation in a wide variety of formats.

\section{Functional Model}

Fig. 4 shows the main functions and information flow involved in the development and production of an aircraft. Management activities, box 'Manage', influence all the downstream functions. 
Development activities, box 'Engineer', are controlled by the output from 'Manage', 'Customer requirements' and 'Industrial strategy'. Development activities include 'Functional Design' and 'Industrial Design'. Both work together, as a single team, to develop product, processes and resources from the conceptual phase to the start of the serial production. The deliverable is an ' $D M U$ ', a complete definition and verification of the virtual manufacturing of the product [8]. All the deviations coming from the shopfloor, in terms of 'Deviations (non conformances, concessions), are inputs to 'Engineer', included in the 'iDMU' and sent to 'Operation'. The final output is an 'As built' iDMU that fits with the real product launch by 'Operation'.

Production activities are represented by the box 'Operation', controlled by the output from 'Manage' and by the output from 'Engineer' 'iDMU'. Operation activities include 'Sustaining', which is in charge of exploit the iDMU, with the help of MES (Manufacturing Execution Systems), to launch 'Shopfloor Documents' to 'Serial production'. The 'Manufacturing Problems' that can be managed without modifying the iDMU, are managed by 'Sustaining'. Any other item affecting the iDMU is derived to 'Engineer' as deviation. The output from 'Operation' is the final physical product that fits $100 \%$ with the 'As built'.

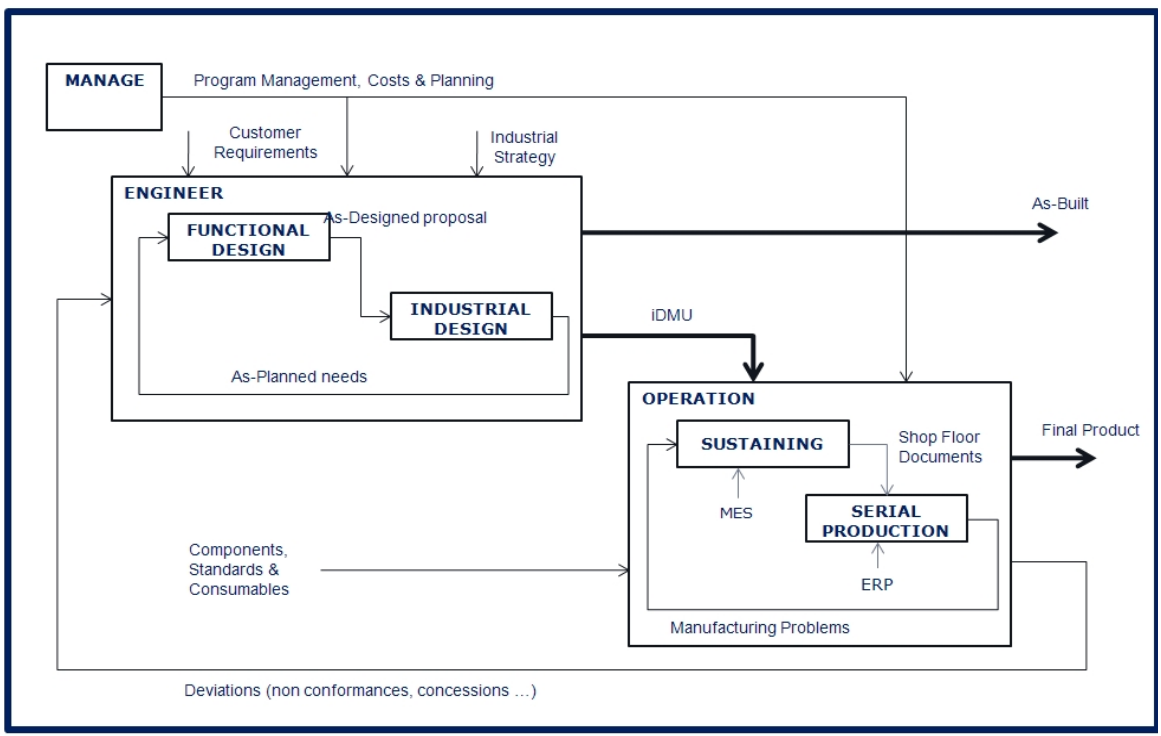

Fig. 4. Collaborative functional model

\section{Digital MockUp (DMU) and Industrial Digital MockUp (iDMU)}

Collaborative Engineering involves a lot of changes in terms of: organization, teams, relationships, skills, methods, procedures, standards, processes, tools and interfaces [4]. It is a business transformation process [5]. One of the key changes is the engineering deliverable, it comprises evolving from the 'product DMU as master' to the ‘iDMU for all' (Fig. 3). 
'DMU as master' is a standard inside Airbus [3]. All the information related to the functional aspects of the product is included in the product DMU, e.g. aspects like 'design in context' and clashes-free product are fully deployed. The DMU is the reference for the product functional definition, and it is built in concurrent engineering taken into account manufacturing constraints.

The 'iDMU for all' is a new concept. It is the main enabler of the Collaborative Engineering approach and provides a common virtual environment for all the aircraft development stakeholders. Functional design and industrial design are part of a single design process where they progress together and influence each other.

The iDMU collects the information related to functional design plus all the information related to industrial design: manufacturing and assembly process, associated resources, industrial means and human resources [8]. All is defined in an integrated environment, where complete and partial simulations are done continuously, and at the end of the design phase, they guarantee a validated solution. Fig. 5 shows an example of a 3D view of an iDMU.

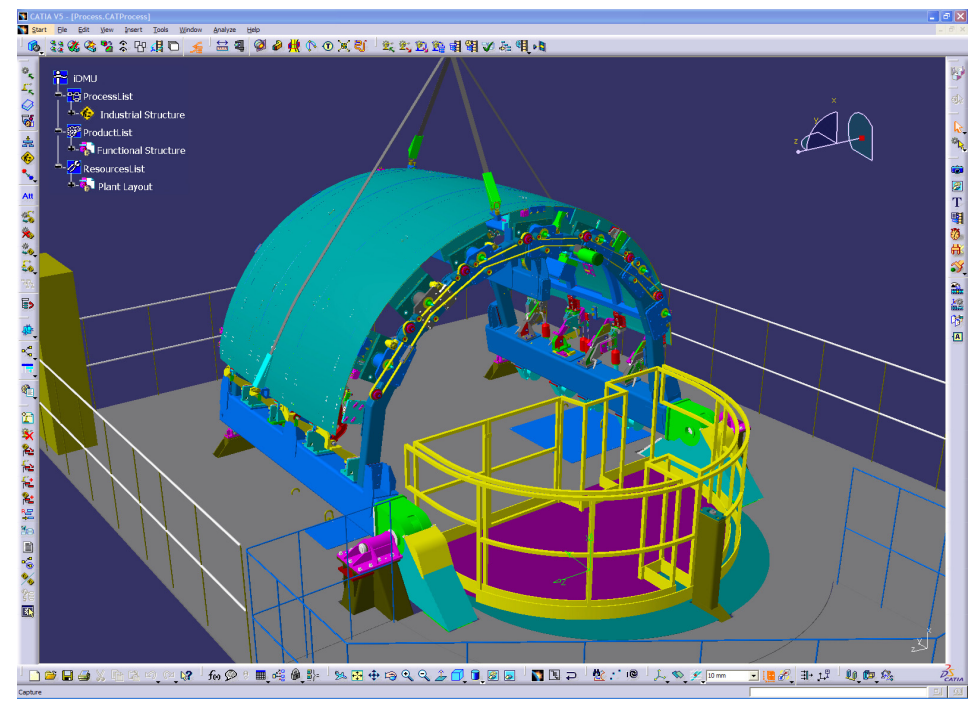

Fig. 5. Fan Cowl industrial Digital MockUp (iDMU) (Not a real iDMU due to confidentiality)

\section{Collaborative Engineering Pilot Project: CALIPSOneo}

CALIPSOneo is an $\mathrm{R}+\mathrm{D}+\mathrm{i}$ pilot project and it was launched early 2013 to support the development, customization and deployment of the PLM tools and processes in Airbus Military. CALIPSOneo is a joined effort that involves Engineering Companies, IT companies, PLM Vendors and Research Centers and Universities. The project runs during 2013 and the first quarter of 2014.

CALIPSOneo makes use of the newest PLM tools. It aims implementing new methods and processes needed to support a collaborative way of designing. It takes as 
input developments from previous projects, related to digital manufacturing techniques implementation [6, 8] and aircraft conceptual design modeling [9]. Similarly to other initiatives reported in literature [10-12], the main results will be definition and modeling of collaborative working procedures, and their implementation into collaborative software tools. The project aims to demonstrate:

- Collaborative Engineering concepts applied to aircraft design and manufacture.

- Capability to generate an iDMU and virtual validation by using it.

- Configuration based on individual specimen.

- To assess the benefits of the iDMU concept when compared to the usual practices for the industrial design.

- Availability of PLM tools to develop and deploy new capabilities.

- Capability to exploit the iDMU to produce advanced shopfloor documentation.

Fig. 6 shows the business model framework where the project is developed. It comprises the frame and conceptual model for all the project activities.

\section{CALIPS Inea}

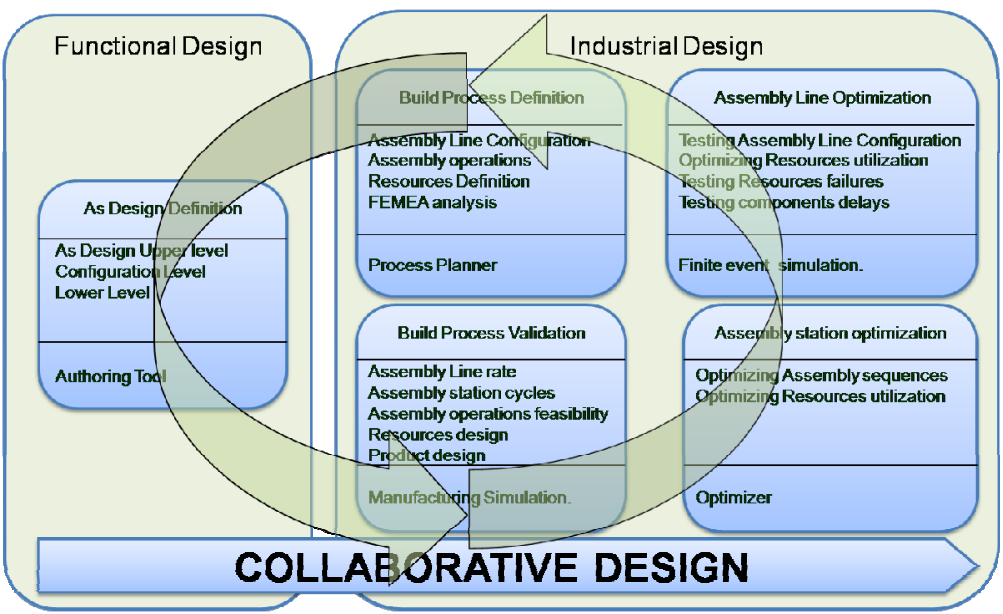

Fig. 6. CALIPSOneo functional diagram

\section{$5 \quad$ Methodology}

The project aims to change the current product design business process to the collaborative paradigm. To achieve it, it is proposed the use of the latest PLM tools to support the new design paradigm. As a consequence, the project required a balanced multiskilled team and a sound methodology to succeed. For that reason, a multi organization project team was created, where Dassault Systèmes, commercial PLM software provider, is the technical advisor, two university research groups are in 
charge of the research work and the methodological support, and two industrial partners are in charge of the development work.

For the systems development methodology, the NDT (Navigational Development Techniques) methodology was selected. NDT was developed by the IWT2 research group (University of Seville) [13]

The project was planned in two main phases, a first phase of analysis and definition and a second phase of implementation and testing of the defined solution.

The NDT methodology uses UML as the documentation technique. In the first phase, requirements defined by suitable use cases and the system data model are defined using a UML based CASE tool (Enterprise Architect). Fig. 7 illustrates a sample of the UML Use cases and data model diagrams.

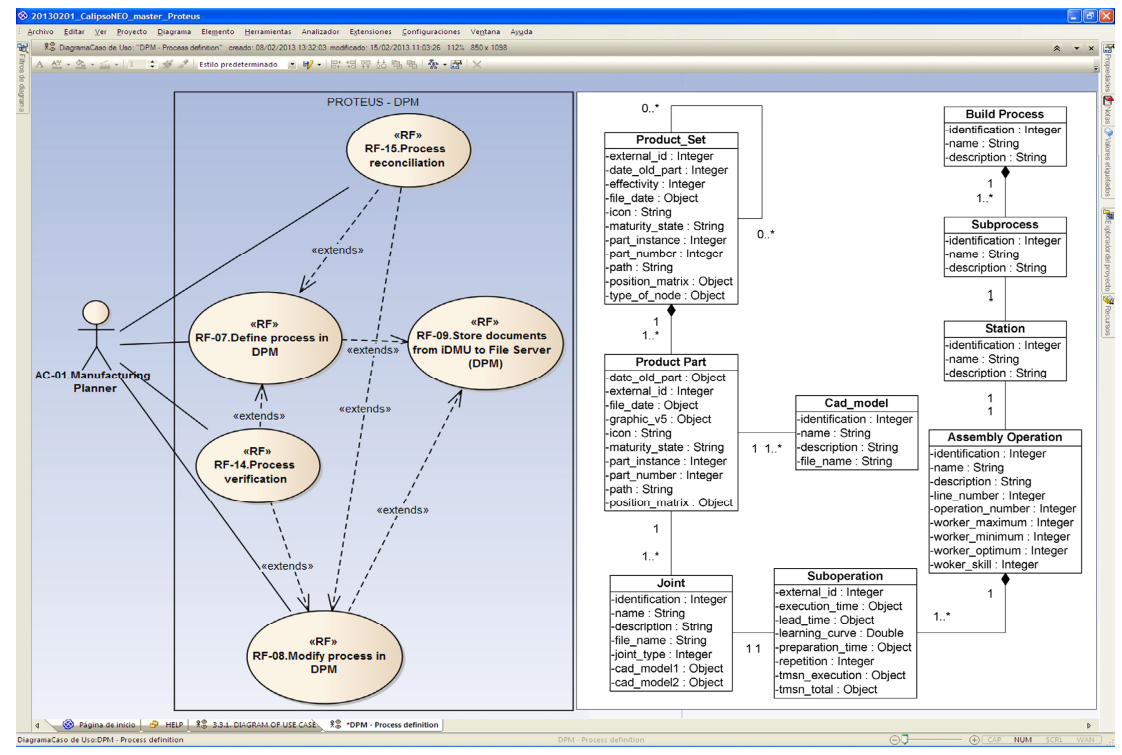

Fig. 7. Use Case \& iDMU structure

A different software application was needed to manage the project documentation and to provide a collaborative environment, for this purpose a web based tool was selected: Alfresco. Alfresco provides a web environment, where all the participants can share all the documents created during the project and it also provides collaborative utilities. Alfresco allows customizing a folder structure for documents, defining review and approving workflows, establishing discussion forums for specific topics and managing the project tasks. In this way, all the documentation and task management were supported by a single tool.

\section{Results and Conclusions}

Collaborative Engineering is a broader approach derived from the previous Concurrent Engineering experiences. The availability of new technologies and the maturity 
of the teams are the key elements in the success of its industrial implementation. During the execution of the CALIPSOneo project, which is still running, several barriers were identified and have to be overcome to implement the Collaborative Engineering approach.

The first issue is the application of the Collaborative Engineering functional model from an organizational and social perspective. It demands the harmonization of the functional design area and the industrial design area, when both are assigned to different departments that are headed by different people. In addition to the organizational issue, the concept of a unique team doing all the design functions has a large set of human relationships implications. Company top management implication is a key factor. CALIPSOneo is helping to make explicit some of the benefits of the collaborative approach in the context of the Company. Extensive literature shows the expected benefits of the collaborative engineering approach, but due to the high impact of the specific organizational and social environment of each company, results must be shown in the company specific context.

The second issue is the complexity of managing a real pilot case in parallel with the development and deployment of the associated PLM tools. The PLM tools are the key enabler in creating and managing the industrial DMU (iDMU). The interoperability between the different commercial software applications and modules used in an industrial project is still an issue. Without the proper integration, the iDMU cannot be created to enable the collaborative approach. For this issue, the adopted solution was to set up a multidisciplinary working team model, where engineers, experts on the industrial design tasks, and PLM experts work altogether conducting industrial and CALIPSOneo R+D+i tasks. Engineers were trained in understanding how PLM tools could help in the industrialization design process. PLM experts were focused on developing specific utilities to facilitate the collaborative work, on customizing the PLM tools and on creating working procedures to create the iDMU.

\section{$7 \quad$ Next Steps}

The next step is consolidating the collaborative process, methods and the associated engineering teams and disseminating the collaborative culture inside Airbus Military and the Extended Enterprise as a standard.

Launch a new $\mathrm{R}+\mathrm{D}+\mathrm{i}$ project, as an extension to the CALIPSOneo project, to identify possible additional issues that may arise and to cover further developments associated to the complexity of larger aerostructures.

Finally, the last step would be the introduction of the collaborative process in a light or medium military transport aircraft, where the Services area will be also considered. The inclusion of MRO design in the iDMU will help to consolidate the collaborative engineering methods, processes and tools along the Company.

Acknowledgements. Authors wish to express their sincere gratitude to colleagues from Airbus Military, Universidad Politécnica de Madrid, Universidad de Sevilla and partners of CALIPSOneo project for their collaboration and contribution to the project. 


\section{References}

1. Aviation News Magazine - AIRBUS A400M, http://www.aviation-news.co.uk/ archive/a400m.html (retrieved in January 2013)

2. Pardessus, T.: Concurrent engineering development and practices for aircraft design at Airbus (Conference). In: Proceedings of the 24th ICAS Meeting, Yokohama (2004)

3. Garbade, R., Dolezal, W.R.: DMU at Airbus - Evolution of the Digital Mock-up (DMU) at Airbus to the Centre of Aircraft Development. In: The Future of Product Development. Proceedings of the 17th CIRP Design Conference, pp. 3-12. Springer (2007)

4. Sriram, D., Logcher, R.: The MIT Dice Project. Computer 26(1), 64-65 (1993)

5. Lu, S.C.-Y., Elmaraghy, W., Schuh, G., Wilhelm, R.: A scientific foundation of collaborative engineering. CIRP Annals - Manufacturing Technology 56(2), 605-634 (2007)

6. Mas Morate, F., Menéndez, J.L., Serván, J., Gómez, A., Ríos, J.: Aerospace Industrial Digital Mock-Up exploitation to generate assembly shop floor documentation. In: Proceedings of 29 International Manufacturing Congress, Belfast (2012)

7. Prasad, B.: Concurrent Engineering Fundamentals. Prentice-Hall PTR, New Jersey (1996)

8. Menendez, J.L., Mas Morate, F., Servan, J., Rios, J.: Virtual verification of an aircraft Final Assembly Line industrialization: an industrial case. Key Engineering Materials. Material Science and Engineering 502, 139-144 (2012)

9. Mas Morate, F., Rios, J., Menendez, J.L., Gomez, A.: A process-oriented approach to modeling the conceptual design of aircraft assembly lines. International Journal of Advanced Manufacturing Technology 62 (2012)

10. Butterfield, J., et al.: Optimization of aircraft fuselage assembly process using digital manufacturing. J. of Comput. Inf. Sci. Eng. 7, 269-275 (2007)

11. Van Wijk, D., et al.: Integrated Design and PLM Applications in Aeronautics Product Development. In: Proc. of the 19th CIRP Design Conf. Competitive Design, Cranfield University (2009)

12. Rouchon, C.: V6 deployment in new business Jet. Top 5 PLM breakthroughs. Proceedings of 3D EXPERIENCE FORUM Europe 2012, Brussels (2012)

13. García-García, J.A., Alba Ortega, M., García-Borgoñon, L., Escalona, M.J.: NDT-Suite: A Model-Based Suite for the Application of NDT. In: Brambilla, M., Tokuda, T., Tolksdorf, R. (eds.) ICWE 2012. LNCS, vol. 7387, pp. 469-472. Springer, Heidelberg (2012) 